\begin{tabular}{|c|c|c|c|}
\hline Eiszeitalter u. Gegenwart & 41 & $\begin{array}{c}70-84 \\
8 \mathrm{Abb} ., 2 \mathrm{Tab} .\end{array}$ & Hannover 1991 \\
\hline
\end{tabular}

\title{
Exoten in der Großsäuger-Fauna des letzten Interglazials von Mitteleuropa
}

\author{
WighaRT VON KOENIGSWALD *)
}

Upper Pleistocene, laste interglacial, large mammal fauna,

Hippopotamus amphibius, Bubalus murrensis, Cervus dama, Dicerorbinus of. megarbinus

Hessia, Baden-Württemberg, Upper Rhine Valley

Kurzfassung: Auf das Vorkommen von Hippopotamus amphibius, Bubalus murrensis, Cervus dama und einem kleinen Nashorn (Dicerorbinus cf. megarbinus) in der letztinterglazialen Fauna der nördlichen Oberrheinebene wird hingewiesen und die ökologischen Voraussetzungen für ihr Vorkommen diskutiert. Im Laufe des Pleistozäns lassen sich für Hippopotamus drei und für Bubalus zwei Einwanderungsphasen unterscheiden, nur im letzten Interglazial kamen beide Arten zusammen vor. Macaca fehlt trotz des regelmäßigen Vorkommens in früheren Interglazialen gerade im letzten. Das deutet darauf hin, daß nicht nur klimatische Faktoren die Einwanderung der Exoten bestimmen und sich die Intensität eines Interglazials nicht an den Einwanderern ablesen läßt.

[Exotic elements in the large mammal fauna of the last interglacial period in the northern Oberrhein area (western Germany)]

Abstract: Hippopotamus amphibius, Bubalus murrensis, Cervus dama, and a small rhinoceros (Dicerorhinus cf. megarbinus) are described as members of the last interglacial fauna from the northern Oberrheinebene (Germany). The ecological significance of their occurrence is being discussed. During the Pleistocene three invasions of Hippopotamus and two of Bubalus can be distinguished stratigraphically. Only during the last interglacial period do both species occur together. In this period Macaca is absent, despite its regular occurrence during earlier interglacial phases. This fact indicates that climate was not only the factor controlling the immigration of exotic species and that the intensity of an interglacial period cannot be deduced from the immigrants.

*) Anschrift des Verfassers: Prof. Dr. Wighart voN KoENIGSWALD, Institut für Paläontologie der Universität Bonn, Nussallee 8, D - 5300 Bonn 1

\section{Einleitung}

Die Funde pleistozäner Knochen in den Schottern der großen Flüsse sind eine wichtige paläontologische Quelle und gehen weit in die vorwissenschaftliche Zeit zurück. In dem hier betrachteten Fundgebiet, der nördlichen Oberrheinebene, hat der Schweizer Arzt WILHELM FABRI im frühen 17. Jahrhundert einen (Mammut-)Oberschenkel in Oppenheim vermessen und ihn einem Riesen zugewiesen (STAMPFLI 1982). Bereits J. H. MERCK (1783) hat in den Knochen von Elefanten und Nashörnern aus den Schottern des Rheins keine Kuriosa mehr gesehen, sondern sie mit moderner Argumentation als Zeugen einer ehemaligen Fauna Mitteleuropas interpretiert.

Obwohl derartiges Faunenmaterial mit dem verstärkten Kiesabbau in den letzten Jahrzehnten in großem Umfang geborgen wurde, blieb das wissenschaftliche Interesse meist gering. Einerseits erwartet man gemeinhin kaum Neuigkeiten aus der Fauna des Jungpleistozäns, und andererseits ist die stratigraphische Zuordnung der Faunenfunde oft nur ungenügend zu bewerkstelligen. Das gilt besonders, wenn die abgebauten Kiese unter dem Grundwasserspiegel liegen und naß mit Saug- und Greifbaggern gefördert werden. Es bleibt dann meist unklar, aus welchen Schotterkörpern das Faunenmaterial stammt. Darüber hinaus sind in Flußschottern Umlagerungen aus älteren Schottern möglich und können nur in besonderen Fällen ausgeschlossen werden.

Andererseits bieten diese Faunenfunde aus den Sanden und Kiesen der großen Flüsse eine wichtige Quelle für die Pleistozänforschung, wie am Beispiel der Fauna des letzten Interglazials gezeigt werden soll, die aus einer Vielzahl von Kiesgruben der nördlichen Oberrheinebene im Raum zwischen Mainz und 


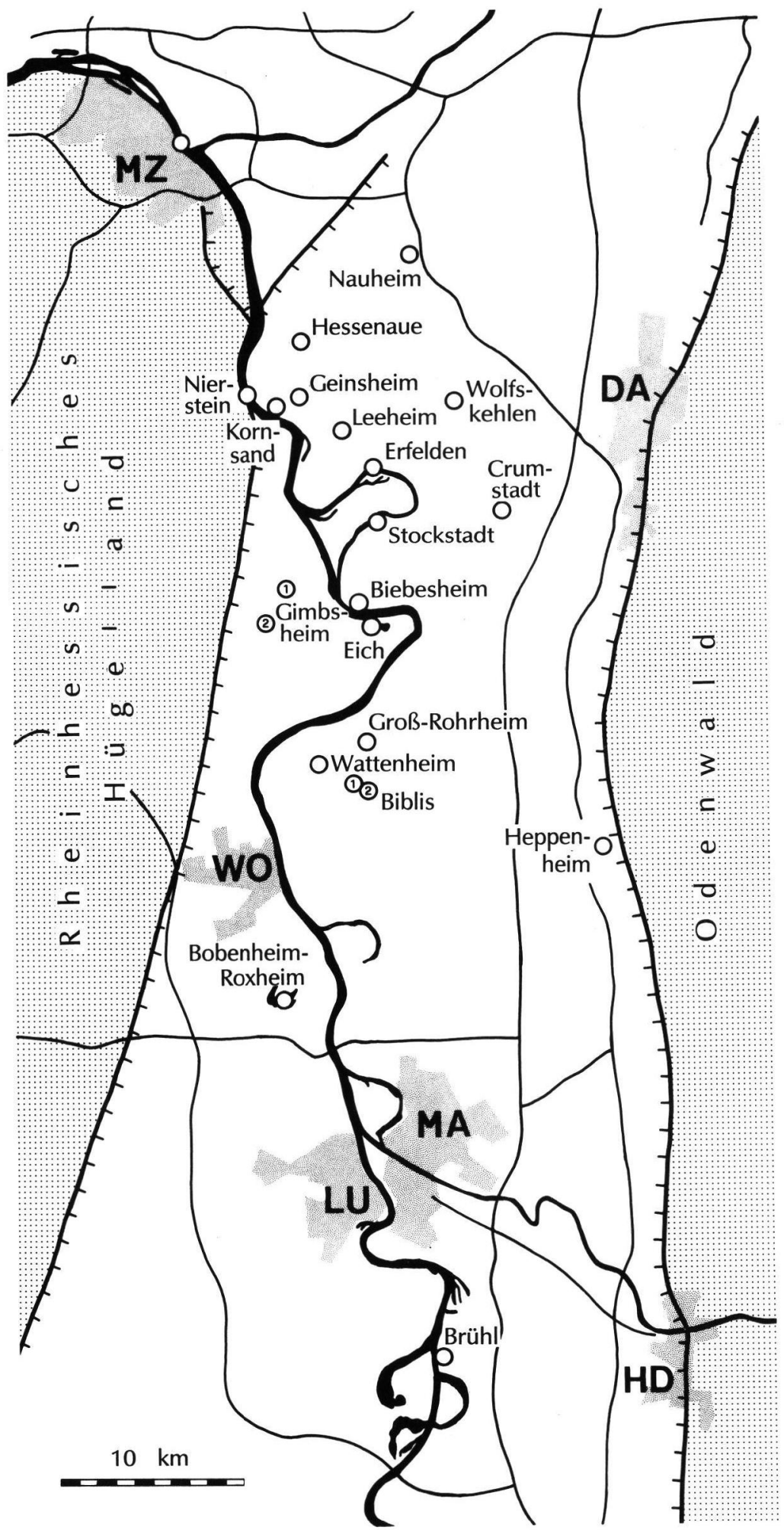

Abb. 1: Karte der nördlichen Oberrheinebene mit den Kiesgruben, die eine umfangreiche jungpleistozäne Säugetierfauna geliefert haben. $(\mathrm{MZ}=$ Mainz, DA = Darmstadt, $\mathrm{WO}=$ Worms, $\mathrm{MA}=$ Mannheim, LU = Ludwigshafen, $\mathrm{HD}=$ Heidelberg). 
Darmstadt im Norden sowie Worms und Heidelberg im Süden aufgesammelt wurde (Abb. 1). Eine kontinuierliche Überwachung der Kiesgruben unter Ausschöpfung aller faßbarer Quellen erlaubte es einer Arbeitsgruppe, die ökologischen Verhältnisse des letzten Interglazials in einem breiten Rahmen zu untersuchen.

Das gemeinsame Vorhaben aus den Jahren 1983-86 stand im Rahmen des nationalen Klimaforschungsprojektes der Bundesrepublik, unter der Leitung von Prof. Dr. Dr. hc. B. Frenzel, „Terrestrische Paläoklimatologie" und wurde vom BMFT über die Akademie der Wissenschaften in Mainz gefördert. Die Mitarbeiter waren: D. SCHWEISS und M. LÖSCHER (Geologie), H.-J. Beug und B. Frenzel (Palynologie), K. U. LeISTIKOW, A. KREUZ und W. H. SCHOCH (Holzanalyse), E. NEUBERT und R. KINZELBACH (Schnecken und Muscheln), R. KLINGER (Holzinsekten) und der Autor (Säugetierpaläontologie und Koordination). Die Ergebnisse wurden in einer gemeinsamen Monographie (KOENIGSWALD [Hrsg.] 1988a) vorgelegt. Die Materialien sind, so weit sie sich nicht in Privatsammlungen befinden, größtenteils im Hessischen Landesmuseum in Darmstadt hinterlegt worden.

Als Exoten werden hier jene Großsäuger verstanden, die zwar in der interglazialen Fauna Mitteleuropas vorkommen, in der holozänen Fauna des Gebietes aber fehlen, obwohl sie nicht ausgestorben sind, sondern mit den gleichen oder sehr nahe verwandten Taxa heute in anderen Gebieten verbreitet sind.
Außer den großen Pflanzenfressern Hippopotamus, Bubalus, Cervus dama und der kleinen Macaca, gehören eigentlich auch die beiden großen Raubtiere Panthera leo spelaea (Höhlenlöwe) und Crocuta crocuta spelaea (Höhlenhyaene) nach der begrifflichen Definition zu den Exoten. Aber im Gegensatz zu den genannten Pflanzenfressern ist das Vorkommen der beiden Carnivora nicht auf die interglazialen $\mathrm{Ab}$ schnitte des Pleistozäns beschränkt. Da sie zu den hier diskutierten ökologischen Bedingungen während des letzten Interglazials nicht beitragen, braucht auf sie nicht näher eingegangen zu werden.

\section{Die Geologischen Verhältnisse in der nördlichen Oberrheinebene}

Die Oberrheinebene ist ein Grabenbruch, dessen Senkungstendenz noch anhält (FAHLBUSCH 1975). Deswegen hat sich besonders im nördlichen Teil keine Terrassentreppe ausgebildet, sondern die jüngeren Sedimente liegen im wesentlichen über den versenkten älteren. Die pleistozänen Sedimente erreichen im Raum zwischen Mainz, Worms, Heidelberg und Darmstadt erhebliche Mächtigkeiten. Während im Westen nur wenige Zehnermeter anstehen, wurden im Osten zwischen Mannheim und Heidelberg $382 \mathrm{~m}$ Quartär erbohrt (BARTZ 1953, 1982). Der Kiesabbau erfaßt davon aber nur den obersten Teil. Besonders im Osten des Gebietes ist in rund 15-25 m Tiefe eine Tonschicht, der „Obere Ton”, weit verbreitet und

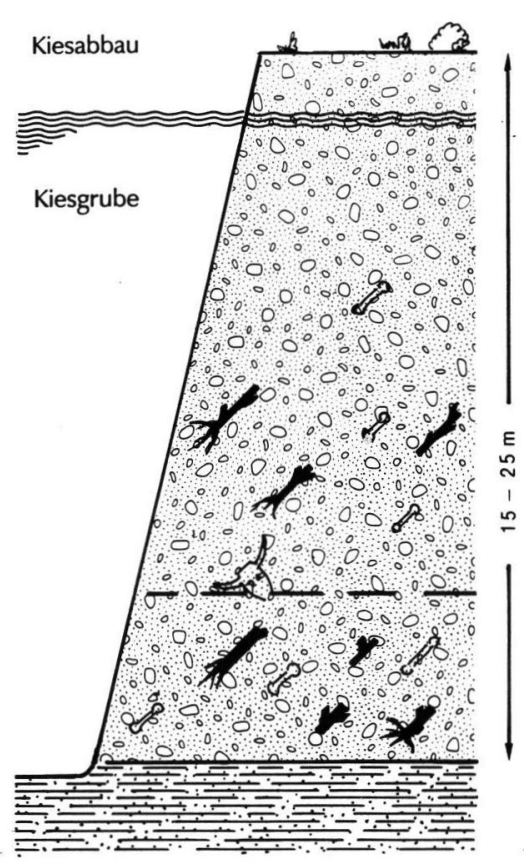

Oberkante der

$\mathrm{Ni}$ ederterras e

Grundwasserspiegel

Sande mit kaltzeitlicher .Fauna und Nadelhölzern (= älterer Abschnitt des letzten Glazials)
Sande mit warmzeitlicher Fauna und vielen Nadel- und Laubhölzern (= letztes Interglazial)

"O b e r e r T o n"
Abb. 2: Schematisches Profil der jungpleistozänen Sedimente in der nördlichen Oberrheinebene, soweit sie vom Kiesabbau betroffen sind. Ein direkter Einblick in das Profil ist nicht möglich, da es fast vollständig unter dem Grundwasserspiegel liegt. 
begrenzt die Sandförderung. Deswegen kann das in den Kiesgruben dieser Region geförderte Knochenmaterial nur aus dem Schichtkomplex oberhalb dieses Oberen Tones stammen.

Der Obere Ton kann sicher als hydrogeologischer wie als stratigraphischer Leithorizont angesehen werden. Allerdings darf seine Feinkörnigkeit keineswegs als klimatisches Indiz gewertet werden. Die während des Pleistozäns anhaltenden tektonischen Bewegungen im Rheinischen Schiefergebirge haben die Transportkraft und das Sedimentationsgeschehen der nördlichen Oberrheinebene weit stärker beeinflußt als klimatische Veränderungen. Deswegen wird der Obere Ton nicht von vornherein dem letzten Interglazial zuge- ordnet, wie er von der ARBEITSGRUPPE (1980) in der hydrologischen Kartierung eingestuft wurde.

Informationen über die Lagerung des Schichtpaketes oberhalb des Oberen Tones (SCHEER 1976, SCHWEISS 1988) stammen aus Bohrungen, aus dem Fördergut der Bagger bei bekannter Arbeitstiefe und aus der intensiven Befragung der Baggerführer während der wiederholten Begehungen der Kiesgruben im Laufe der Jahre. LÖSCHER $(1981,1988)$ konnte im Heidelberger-Raum feststellen, daß die Baggerführer in der Regel recht gut wissen, in welchen Tiefen sich der Sandabbau etwa durch das vermehrte Auftreten von eingelagerten Baumstämmen für sie erschwert. Theoretisch kann man nur maximale Tiefenangaben für
Klimatisch indifferente Arten

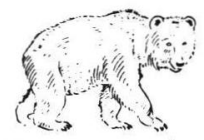

Ursus spelaeus

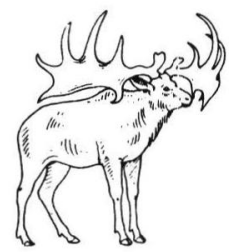

Megaloceros giganteus

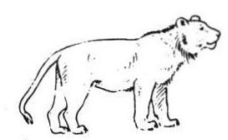

Panthera spelaea

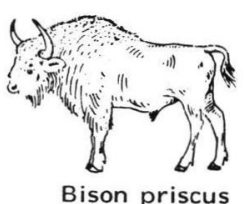

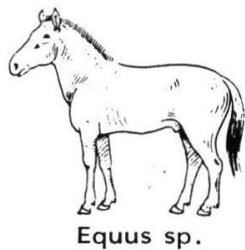

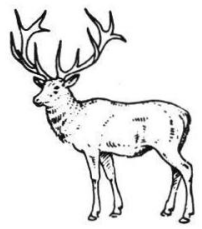

Cervus elaphus

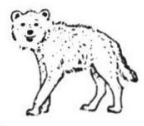

Crocuta spelaea

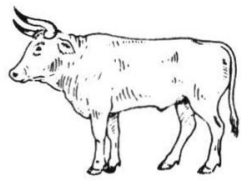

Bos primigenius

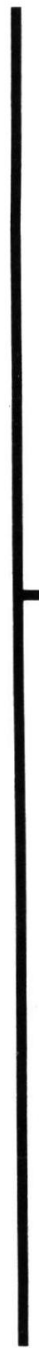

\section{Klimaindikatoren des Glazials}

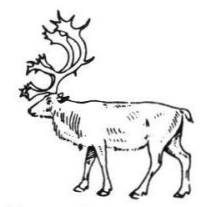

Rangifer tarandus
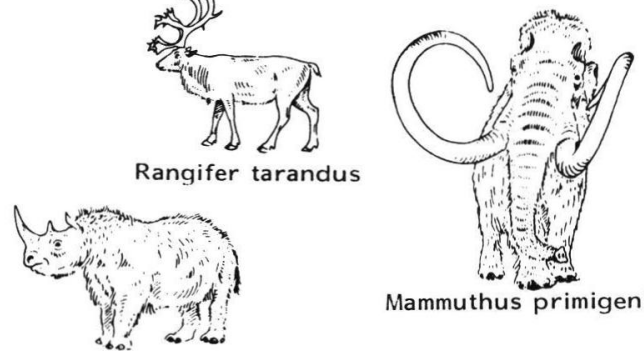

Mammuthus primigenius

Coelodonta antiquitatis

Klimaindikatoren des letzten Interglazials

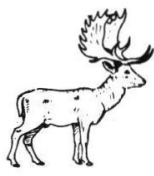

Cervus dama

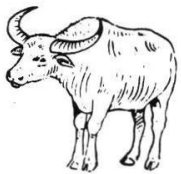

Bubalus murrensis
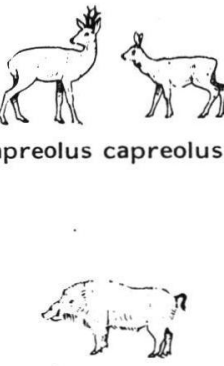

Capreolus capreolus

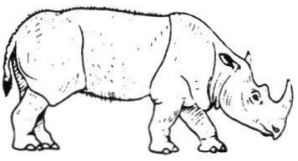

Dicerorhinus kirchbergensis

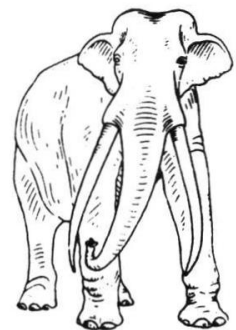

Elephas (P.) antiquus

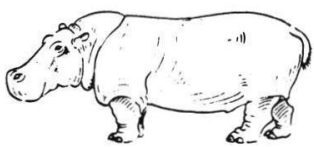

Hippopotamus amphibius

Abb. 3: Jungpleistozäne Großsäugerfauna aus der nördlichen Oberrheinebene, nach den klimatischen Ansprüchen in drei Gruppen aufgeteilt. (Rekonstruktionen aus THENIUS 1962). 
Knochenfunde geben, aber Baggerführer merken sehr deutlich, ob sie aus dem Anstehenden oder dem Nachbruch fördern. Deswegen kann durchaus eine schematische Gliederung gegeben werden (Abb. 2).

In den weitgehend fein- bis mittelsandigen Abfolgen konnte im oberen Teil eine kaltzeitliche Fauna mit Mammuthus primigenius, Coelodonta antiquitatis und Rangifer tarandus festgestellt werden. Stellenweise wurden Stämme von Nadelhölzern aus diesem Tiefenbereich gefördert (s. a. LÖSCHER et al. 1980. LÖSCHER 1988).
Im unteren Profilabschnitt dicht über dem Oberen Ton nehmen in fast allen Kiesgruben die Holzfunde zu. Neben Nadelhölzern treten hier tief schwarze Stämme von Eichen hervor, die einen Durchmesser von $50-60 \mathrm{~cm}$ oder auch mehr erreichen. Das Holz kann von den Baggerrosten abgesammelt werden. Im Rahmen des Projektes wurde es durch KREUZ \& LEISTIKOW (1988) sowie durch SCHOCH (1988) bearbeitet.

Aus dem Auftreten der Holzarten eines Eichenmischwaldes kann geschlossen werden, daß unter dem durch die Fauna als kaltzeitlich gekennzeichneten

Tabelle 1: Nachweis jungpleistozäner Großsäuger in den wichtigsten Kiesgruben der nördlichen Oberrheinebene (vgl. Abb. 1). Die Gliederung in die ökologischen Gruppen 1 bis 3 entspricht der Abb. 3.

\begin{tabular}{|c|c|c|c|c|c|c|c|c|c|c|c|c|c|c|c|c|c|c|c|c|c|}
\hline & & $\begin{array}{c}\text { upp } \\
1\end{array}$ & & & & & & $\begin{array}{r}\text { Gru } \\
2\end{array}$ & $\begin{array}{l}\text { ppe } \\
\text { ? }\end{array}$ & & & & & & & & & & & & \\
\hline & 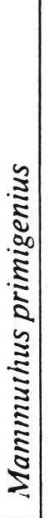 & 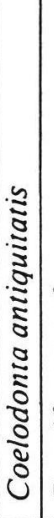 & $\begin{array}{l}5 \\
3 \\
0 \\
5 \\
5 \\
0 \\
\vdots \\
0 \\
0 \\
5 \\
0 \\
0\end{array}$ & 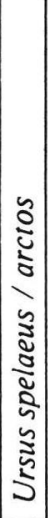 & 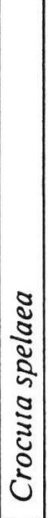 & 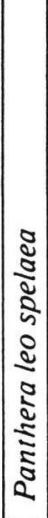 & 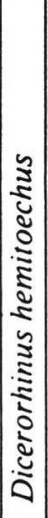 & 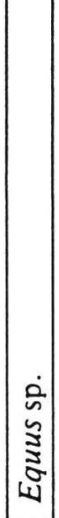 & 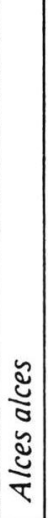 & 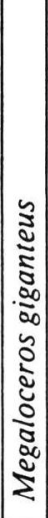 & 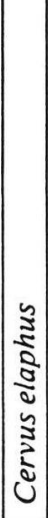 & 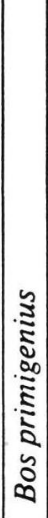 & 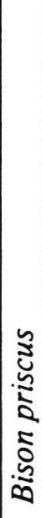 & 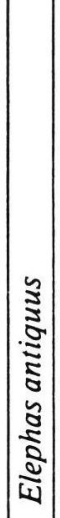 & 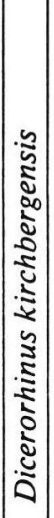 & 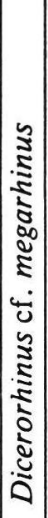 & 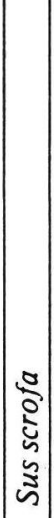 & 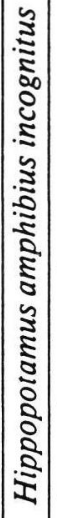 & 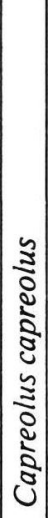 & 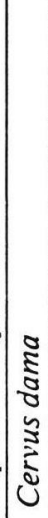 & $\begin{array}{l}5 \\
5 \\
5 \\
5 \\
5 \\
5 \\
5\end{array}$ \\
\hline $\begin{array}{l}\text { Biblis } \\
\text { Biebesheim } \\
\text { Bobenheim-Roxheim } \\
\text { Brühl 1 b. Mannheim } \\
\text { Brühl } 2 \text { b. Mannheim } \\
\text { Crumstadt } 1 \\
\text { Eich } \\
\text { Erfelden } \\
\text { Geinsheim } \\
\text { Gernsheim } \\
\text { Gimbsheim } 2 \\
\text { Groß-Rohrheim } \\
\text { Hessenaue } \\
\text { Huttenheim } \\
\text { Kornsand } \\
\text { Leeheim } \\
\text { Mainz } \\
\text { Nauhcim } \\
\text { Stockstadt } \\
\text { Wattenheim } \\
\text { Wolfskehlen }\end{array}$ & $\begin{array}{l}\bullet \\
\bullet \\
\bullet \\
\bullet \\
\bullet \\
\bullet \\
\bullet \\
\bullet \\
\bullet \\
\bullet \\
\bullet \\
\bullet \\
\bullet\end{array}$ & $\bullet$ & $\begin{array}{l}\bullet \\
\bullet \\
\bullet \\
\bullet \\
\bullet\end{array}$ & $\mid \begin{array}{l}\bullet \\
\bullet \\
\bullet \\
\bullet \\
\bullet\end{array}$ & • & $\bullet$ & $\begin{array}{l}\bullet \\
\bullet \\
\bullet\end{array}$ & $\begin{array}{l}\bullet \\
\bullet \\
\bullet \\
\bullet \\
\bullet \\
\bullet \\
\bullet \\
\bullet \\
\bullet \\
\bullet \\
\bullet \\
\bullet \\
\bullet \\
\bullet \\
\bullet \\
\bullet \\
\bullet \\
\bullet\end{array}$ & $\begin{array}{l}\bullet \\
\bullet \\
\bullet \\
\bullet \\
\bullet\end{array}$ & $\begin{array}{l}\bullet \\
\bullet \\
\bullet \\
\bullet \\
\bullet \\
\bullet \\
\bullet \\
\bullet \\
\bullet \\
\bullet \\
\bullet \\
\bullet\end{array}$ & $\begin{array}{l}\bullet \\
\bullet \\
\bullet \\
\bullet \\
\bullet \\
\bullet \\
\bullet \\
\bullet \\
\bullet \\
\bullet\end{array}$ & $\begin{array}{l}\bullet \\
\bullet \\
\bullet \\
\bullet \\
\bullet \\
\bullet \\
\bullet \\
\bullet \\
\bullet \\
\bullet\end{array}$ & $\begin{array}{l}\bullet \\
\bullet \\
\bullet \\
\bullet \\
\bullet \\
\bullet \\
\bullet \\
\bullet \\
\bullet \\
\bullet \\
\bullet\end{array}$ & $\begin{array}{ll}\bullet \\
\bullet \\
\bullet \\
\bullet \\
\bullet \\
\bullet \\
\bullet \\
\bullet \\
\bullet \\
\bullet \\
\bullet \\
\bullet \\
\bullet \\
\bullet \\
\bullet\end{array}$ & • & • & $\begin{array}{l}\bullet \\
\bullet \\
\bullet \\
\bullet\end{array}$ & $\begin{array}{l}\bullet \\
\bullet \\
\bullet \\
\bullet \\
\bullet\end{array}$ & • & • & $\bullet$ \\
\hline
\end{tabular}


Sand des oberen Profilabschnittes auch die Ablagerungen einer Warmzeit vom Abbau erfaßt werden. Faunenfunde, wie die von Elephas (P.) antiquus, bestätigen dieses Bild. Der Übergang von der Warmzeit zur folgenden Kaltzeit wird aber von keinem Wechsel in den Sedimenten begleitet und kann nicht näher aufgeschlüsselt werden.

Oft lassen sich die Faunenfunde aus dem Baggergut keinem bestimmten Profilabschnitt zuordnen. Die Gesamtfauna setzt sich sowohl aus typisch kaltzeitlichen und warmzeitlichen Arten und einer größeren Zahl von Formen zusammen, denen keine klimatische Präferenz zugeordnet werden kann (Abb. 3; Tab. 1).

\section{Das Faunenspektrum}

Das Spektrum der Säugetierarten ist zunächst durch die Größe der Knochen bestimmt. Denn nur das Material, das auf dem Grobrost des Baggers gefangen wird oder im Überkorn abgesiebt wird, hat eine Chance, gefunden zu werden. Daher fehlen die sowohl stratigraphisch wie ökologisch besonders aussagefähigen Kleinsäuger. Gefunden wurden folgende Arten:

Canis lupus
Crocuta spelaea
Patbera leo spelaea
Ursus spelaeus
Ursus arctos
Elephas (Palaeoloxodon) antiquus
Mammuthus primigenius
Equus sp.
Dicerorhinus kirchbergensis
Dicerorbinus hemitoechus
Dicerorhinus cf. megarhinus
Coelodonta antiquitatis
Sus scrofa
Hippopotamus amphibius incognitus
Cervus elaphus
Cervus (Dama) dama
Capreolus
Rangifer tarandus
Megaloceros giganteus
Alces sp.
Bison priscus
Bos primigenius
Bubalus murrensis

Nicht unbedingt nach der Tiefenlage aber nach den ökologischen Ansprüchen können diese Arten in drei Gruppen aufgeteilt werden (Abb. 3). Als typisch kaltzeitlich werden Mammuthus primigenius, Coelodonta antiquitatis und Rangifer tarandus gewertet. Als warmzeitlich gelten Elephas (P.) antiquitatis, Dicerorhinus kirchbergensis, Sus scrofa, Hippopotamus am- phibius, Cervus dama, Capreolus capreolus, Bubalus murrensis, während die übrigen Arten keine strenge ökologische Bindung aufweisen. Die Verteilung der Faunenfunde auf die einzelnen Kiesgruben ergibt sich aus Tabelle 1.

In der Gruppe der warmzeitlichen Formen muß die Zusammengehörigkeit zu einer gleichzeitigen Fauna besonders streng überprüft werden, denn fluviatile Sedimente lassen stets eine gewisse Umlagerung erwarten.

Bei dem Vorkommen von Capreolus capreolus und Sus scrofa, muß die Einmischung von subfossilem Material aus dem Holozän, von Fall zu Fall, aufgrund der Erhaltung ausgeschlossen werden. Funde von Hippopotamus amphibius in den Rheinschottern wurden von ADAM (1965) dem Mosbachium zugeordnet und in jüngeren Schichten als umgelagert angesehen. Der Fund von mehreren Eckzähnen mit vorzüglich erhaltenen Spitzen sowie der von zwei zusammengehörigen Wirbeln in der Kiesgrube Wattenheim schließen eine Umlagerung aus älteren Sedimenten aus. Für einen Schädel von Bubalus murrensis haben NEUFER \& IGEL (1983) eine Umlagerung postulieren müssen, um ein akzeptables stratigraphisches Alter zu begründen. Die Erhaltung dieses Schädels, aber auch aller anderen Schädel von Bubalus murrensis, spricht gegen eine Umlagerung, weil die Spitzen und Kanten der Hornzapfen in keinem Fall abgerollt waren. Die zum Teil erheblichen Zerstörungen, die durch die Förderung mit dem Greifbagger entstanden sind, lassen sich deutlich von möglichen Abrollspuren durch Umlagerungen unterscheiden. Gegen eine Umlagerung spricht neben der Erhaltung auch die Fundverteilung. Immerhin 4 räumlich weit auseinanderliegende Gruben (Eich, Huttenheim, Leeheim und Stockstadt) haben gleichzeitig Reste von Hippopotamus und von Bubalus geliefert (Tab. 1). Die relative Häufigkeit von beiden Gattungen im Gebiet und die Konzentration von Hippopotamus in Groß-Rohrheim macht eine Umlagerung besonders unwahrscheinlich, weil diese eher zu einer Vereinzelung führen würde.

Eine synchrone Umlagerung, also ein Transport von Einzelknochen oder mehr oder weniger frischen Tierleichen innerhalb des Stromsystems kann nicht ausgeschlossen werden. Aber bei der Orographie des Oberrheingebietes führt das zu keinen wesentlichen Fehlern in der ökologischen Interpretation. Es gelang durch die regelmäßige Überwachung der Bagger und die Zusammenarbeit mit Privatsammlern neben Einzelknochen auch größere Teile zusammengehöriger Skelette zu bergen, besonders von den großen Boviden (MARTIN 1987). Als ganz ungewöhnlicher Fund ist hier ein weitgehend vollständiges Skelett von Elephas (P.) antiquus aus Crumstadt bei Darmstadt 
zu nennen, das aus etwa 13 m Tiefe mit dem Saugbagger gefördert wurde (KOENIGSWALD 1986a). Diese Funde machen deutlich, daß synchrone Umlagerungen zwar möglich, aber keineswegs allgemein anzunehmen sind.

Aus diesen Gründen werden die rein warmzeitlichen Arten als eine natürliche Faunenassoziation aus einem einzigen Interglazial angenommen. Wegen der fehlenden Fundbeobachtung im Sediment kann allerdings eine potentiell vorhandene feinstratigraphische Abfolge nicht ausgeschlossen werden.

\section{Die stratigraphische Zuordnung}

Die hier zu diskutierende warmzeitliche Fauna liegt weit außerhalb des Moränengebietes und kann damit nicht unmittelbar einem der Interglaziale zugeordnet werden. Die Fauna wird zumindest von einer kaltzeitlichen Fauna mit Mammutbus primigenius, Coelodonta antiquitatis und Rangifer tarandus überlagert. $\mathrm{Da}$ es sich um ein Senkungsgebiet ohne erhebliche Erosionen handelt, ist nach der Lagerung eine Einstufung in das letzte Interglazial wahrscheinlich. Faunen aus den unterlagernden Schichten sind nicht bekannt. Im Gegensatz zu den jüngst im Leipziger Raum erfolgten Untersuchungen zu jungpleistozänen Interglazialvorkommen (EISSMANN 1990) war es in der nördlichen Oberrheinebene nicht möglich, auch nur eine einigermaßen durchgehende Sequenz von Pollenproben zu gewinnen, weil die kalkreichen Feinsande in der Regel keine Pollenüberlieferung erlauben. Beug (1988) hat eine Reihe von isolierten Proben untersucht, konnte aber nur in Ausnahmen volle interglaziale Verhältnisse antreffen. Die Probe aus dem Hornzapfen eines Wasserbüffels von Stockstadt, die FrENZEL (1988) untersucht hat, ergab dagegen ein voll interglaziales Florenbild. Die Sequenz der einwandernden Baumarten konnte aus den Proben nicht rekonstruiert werden, woduch eine definitive Zuordnung zu einem bestimmten Interglazial aufgrund der Pollenanalyse nicht möglich ist.

Absolute Altersbestimmungen an Torfen und Knochen bzw. Zähnen ergaben widersprüchliche Daten (Koenigswald \& Beug 1988), die z. T. in den Zeitbereich des letzten Interglazials fallen, $z$. T. aber auch ein früheres Interglazial nicht ausschließen.

Die angetroffenen Faunenelemente haben keine so hohen stratigraphischen Leitwerte, daß sich die Altersstellung ohne weitere Argumentation ergeben würde. Allerdings treten zwei Arten nur selten im mittelund westeuropäischen Pleistozän auf, so daß hier eine stratigraphische Korrelation möglich ist. Bubalus murrensis ist außerhalb des Rheingebietes nur zwei- mal belegt, nämlich im Interglazial von Steinheim an der Murr (BERCKHEMER 1927) und Schönbeck an der Elbe (SCHERZ 1937). Diese Vorkommen werden mit dem Holstein korreliert, wenngleich hierfür der direkte florenkundliche Nachweis aussteht. Wegen dieses Vorkommens wurden die ersten Funde von Bubalus aus dem Rheingebiet auch dem Holstein zugeordnet (FRANZEN \& KOENIGSWALD 1979), obwohl FRANZEN (1978) zunächst aufgrund der Lagerungsverhältnisse ein letztinterglaziales Alter angenommen hatte. Funde von Hippopotamus wurden von ADAM (1965) stets dem Mosbachium zugeordnet, weil ein jüngeres Vorkommen dieser Gattung in Mitteleuropa unbekannt war. LÖSCHER (1981) stellte aufgrund der Lagerungsverhältnisse die ersten Funde von Hippopotamus in das letzte Interglazial. In England gehört Hippopotamus amphibius incognitus sehr regelmäßig zum Faunenbestand des letzten Interglazials (Ipswichian) (SUTCLIFFE 1959; STUART 1986), kommt aber nicht in den vorangehenden Warmzeiten vor. Es ist zu erwarten, daß diese Hippopotamus-Populationen der Britischen Inseln über den Kontinent entlang der großen Flüsse eingewandert sind. Mit diesem faunengeographischen Argument haben KoENIGSWALD \& LÖSCHER (1982) die Hippopotamus-Funde aus der nördlichen Oberrheinebene ebenfalls dem letzten Interglazial zugeordnet, weil eine Umlagerung aus dem Mosbachium wegen der Erhaltung ausgeschlossen werden konnte und die geologische Lagerung ein höheres Alter als das letzte Interglazial unwahrscheinlich machte.

\section{Die Exoten}

\subsection{Hippopotamus amphibius incognitus}

Einzelne Hippopotamus-Funde aus der Oberrheinebene sind in der älteren Literatur erwähnt. Die gezielten Aufsammlungen der letzten Jahre erbrachten mehr als 30 Belege aus 10 Gruben (KOENIGSWALD 1988), wobei die Stücke in Privatsammlungen nur unvollständig erfaßt werden konnten. Es handelt sich im wesentlichen um die auffallenden Eckzähne (Abb. 4) und Molaren. Skelettknochen sind seltener aufgesammelt worden. Aufgrund der großräumigen Verteilung der Funde und deren guter Erhaltung läßt sich eine Umlagerung aus älteren Sedimenten auschließen.

Das Auftreten von Hippopotamus im letzten Interglazial von Mitteleuropa war zu postulieren, da diese Gattung in England in diesem Zeitabschnitt weit verbreitet ist. Die möglichen Einwanderungswege führen vom Mittelmeergebiet über die großen Flußsysteme nach Mittel- und Westeuropa. Dabei kommt die Donau aus klimageographischen Gründen weniger in Betracht als die Rhone. Von dort konnte sich 


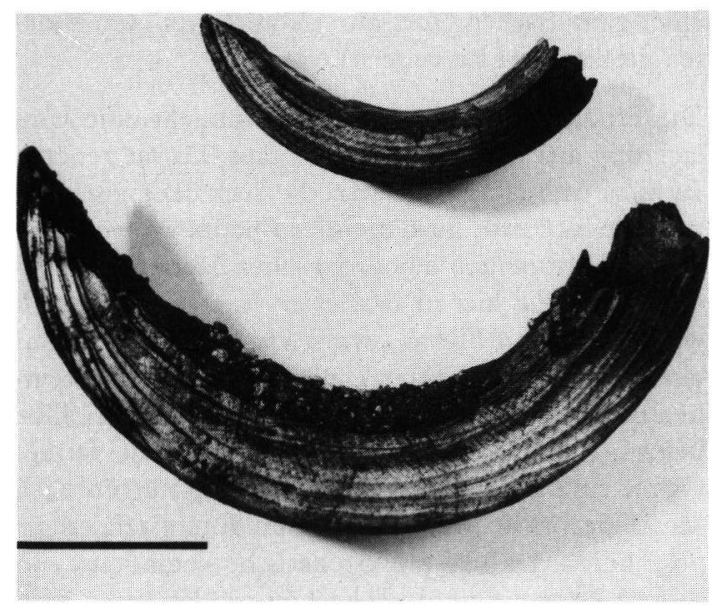

Abb. 4: Untere Eckzähne verschiedener Individuen von Hippopotamus amphibius incognitus.

Der kleine Eckzahn (oben) stammt aus Groß-Rohrheim (Hess. Landesmus. Darmstadt HLMD-RS 2900), der größere aus Wattenheim (Hess. Landesmus. Darmstadt HLMD-RS 2401). Maßstab $=10 \mathrm{~cm}$.

Hippopotamus über die Loire, die Seine, aber wie durch die Funde nun nachgewiesen, auch über den Rhein weiter nach Norden ausbreiten. Inzwischen sind auch Funde aus ähnlicher geologischer Situation vom Niederrhein und aus Holland bekannt geworden. Bislang fehlen die Funde von Hippopotamus östlich der Rhone-Rheinlinie für das letzte Interglazial. Es kann sich dabei zwar noch um eine Fundlücke handeln, aber die derzeitig bekannte Verbreitung paßt sehr gut zu dem atlantischen Klimaeinfluß, der eine ökologische Voraussetzung für das Auftreten von Hippopotamus in Mitteleuropa sein dürfte (s. Kap. 6).

Außer dem Vorkommen von Hippopotamus während des letzten Interglazials lassen sich bisher nur zwei frühere Einwanderungsphasen von Hippopotamus in Mitteleuropa nachweisen. Das früheste Vorkommen ist durch umfangreiche Funde aus Meiningen-Untermaßfeld [Thüringen, D] (KAHLKE, H. D. 1982; KAHLKE R. D. 1985, 1990) belegt. Dieser Fundort ist nach der Säugetierfauna in das Altpleistozän zu stellen. In den paläomagnetischen Befunden ist der Jaramillo-Event zu fassen (WIEGANK 1982). In übereinstimmender paläomagnetischer Position wurde in der Grube Kärlich im Neuwieder-Becken [Rheinland, D] ein Zahnfragment vom Flußpferd im basalen Schotter (A) gefunden (KOLFSCHOTEN 1990).

In den jüngeren Faunen des Altpleistozäns und denen des früheren Mittelpleistozäns scheint Hippopotamus $\mathrm{zu}$ fehlen. Das Cromer sensu stricto mit den klassischen Mimomys savini-Faunen von West-Runton [East Anglia, GB], Voigtstedt und Süssenborn [Thüringen, D], enthält kein Hippopotamus. Allerdings tritt Hippopotamus dann in den älteren ArvicolaFaunen des Mosbachiums von Mauer bei Heidelberg [Baden, D] und Mosbach bei Wiesbaden [Hessen, D] zusammen mit Elephas (P.) antiquus auf. Sie werden einem jüngeren Interglazial als dem Cromerium sensu stricto zugerechnet. Durch den Nachweis von Arvicola im niederländischen Cromer IV in Noordbergum [Groningen, NL] ist sichergestellt, daß diese zweite Einwanderung zeitlich älter ist als die ElsterVereisung. Hippopotamus-Funde aus East-Anglia
Abb. 5: Schädelfragment von Bubalus murrensis mit den weitgeschwungenen, oben abgeplatteten Hornzapfen und dem weit nach hinten überstehenden Hinterhaupt. Der Schädel wurde in Wolfskehlen gefunden (Felsbergmuseum, Beedenkirchen). $\mathrm{Maßstab}=10 \mathrm{~cm}$.

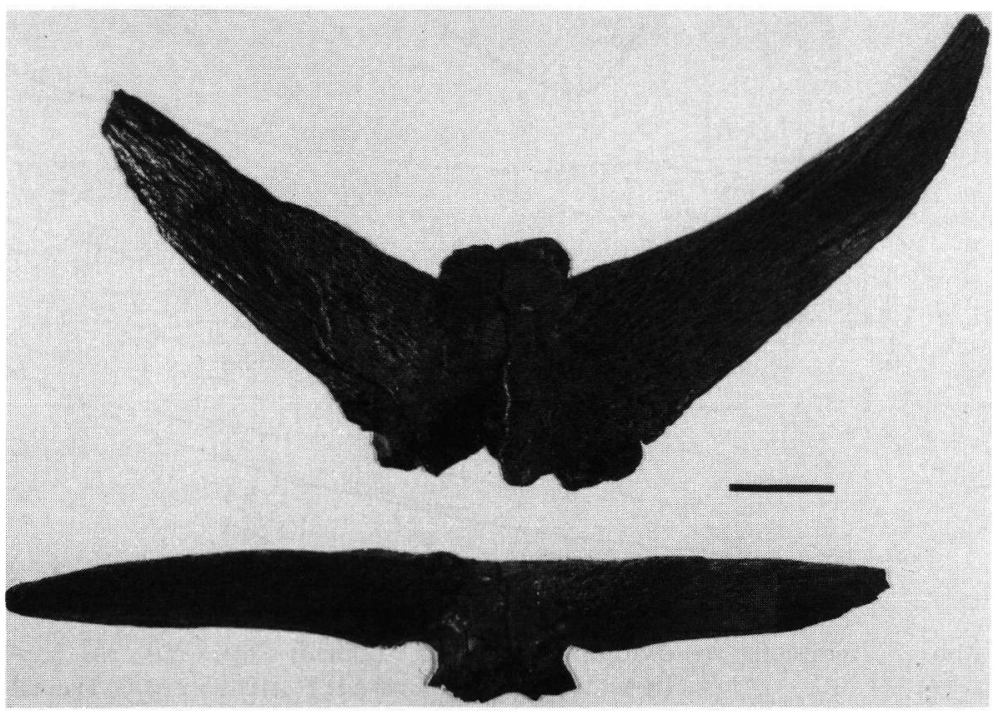


[GB], die STUART (1986) den Pollenzonen III b oder IV des Cromerium zugewiesen hat, können sehr wohl auch zu den älteren Arvicola-Faunen eines jüngeren Interglazials gehören.

Die zeitliche Einstufung der Vorkommen von Hippopotamus in Würzburg-Schalksberg [Franken, D] (RUTTE 1968, KALKE 1989) und besonders Jockgrim [Pfalz, D] (Kuss 1957), die dem Cromerium sensu lato zugerechnet werden, bleibt unsicher, weil bisher keine Kleinsäuger gefunden wurden, die eine feinere Einstufung dieser Fundstellen ermöglichen.

Für das jüngere Mittelpleistozän gibt es keine Belege für Hippopotamus im Raum nördlich der Alpen. Im Hoxnian Englands tritt im Gegensatz zum Ipswichian kein Hippopotamus auf (SUTCLIFFE 1959, 1985; STUART 1986).

\subsection{Bubalus murrensis}

In der nördlichen Oberrheinebene wurden in den letzten 20 Jahren etwa 10 Schädel- und Hornzapfenfragmente von Bubalus murrensis identifiziert (Abb. 5). Bislang ist es nicht gelungen, im postcranialen Material aus den Kiesgruben auch den Wasserbüffel zu erkennen. Selbst die postcranialen Skelettelemente der häufig vertretenen großen Boviden, Bos und Bison, sind nur schwer und keineswegs immer eindeutig zu unterscheiden (MARTIN 1987). Dagegen lassen sich Schädelfragmente von Bubalus an dem charakteristisch dreieckigen Querschnitt der Hornzapfen mit einer ganz flachen Oberseite (Abb. 6) von den anderen großen Boviden unterscheiden.

Die Erhaltung der Bubalus-Reste schließt eine Umlagerung aus älteren Sedimenten aus. Damit gehören Bubalus und Hippopotamus, die auch ökologisch gut zusammenpassen, auch wenn sie heute auf verschiedenen Kontinenten leben, zu einer Faunenvergesellschaftung, die hier in das letzte Interglazial gestellt wird. Bisher war Bubalus murrensis allerdings nur aus einem älteren Interglazial, nämlich dem von Steinheim an der Murr und von Schönebeck an der Elbe belegt, das mit dem Holstein gleichgesetzt wurde. Damit sind für Bubalus zwei Einwanderungen nach Mitteleuropa in unterschiedlichen Interglazialen zu postulieren. Da eine phylogenetische Veränderung in diesem Zeitraum nicht beobachtet werden kann, wird der gleiche Artnamen benutzt.

Vom rezenten indischen Wasserbüffel, Bubalus arnee, unterscheidet sich Bubalus murrensis erheblich. Die Stellung der Hornzapfen und das weit nach hinten überragende Hinterhaupt sind eindeutig primitive Merkmale, die beim indischen Wasserbüffel mit den Unterarten Bubalus arnee palaeokerabau und Bubalus arnee palaeoindicus schon seit dem Altpleistozän weiterentwickelt sind. Damit belegt Bubalus arnee eine andere Entwicklungslinie. Da die Bubalus-Arten des Pleistozäns von China eine ähnliche Merkmalsausprägung wie Bubalus murrensis aufweisen, dürften sie trotz der damit verbundenen geographischen Probleme einander näher stehen (KOENIGSWALD 1986b).

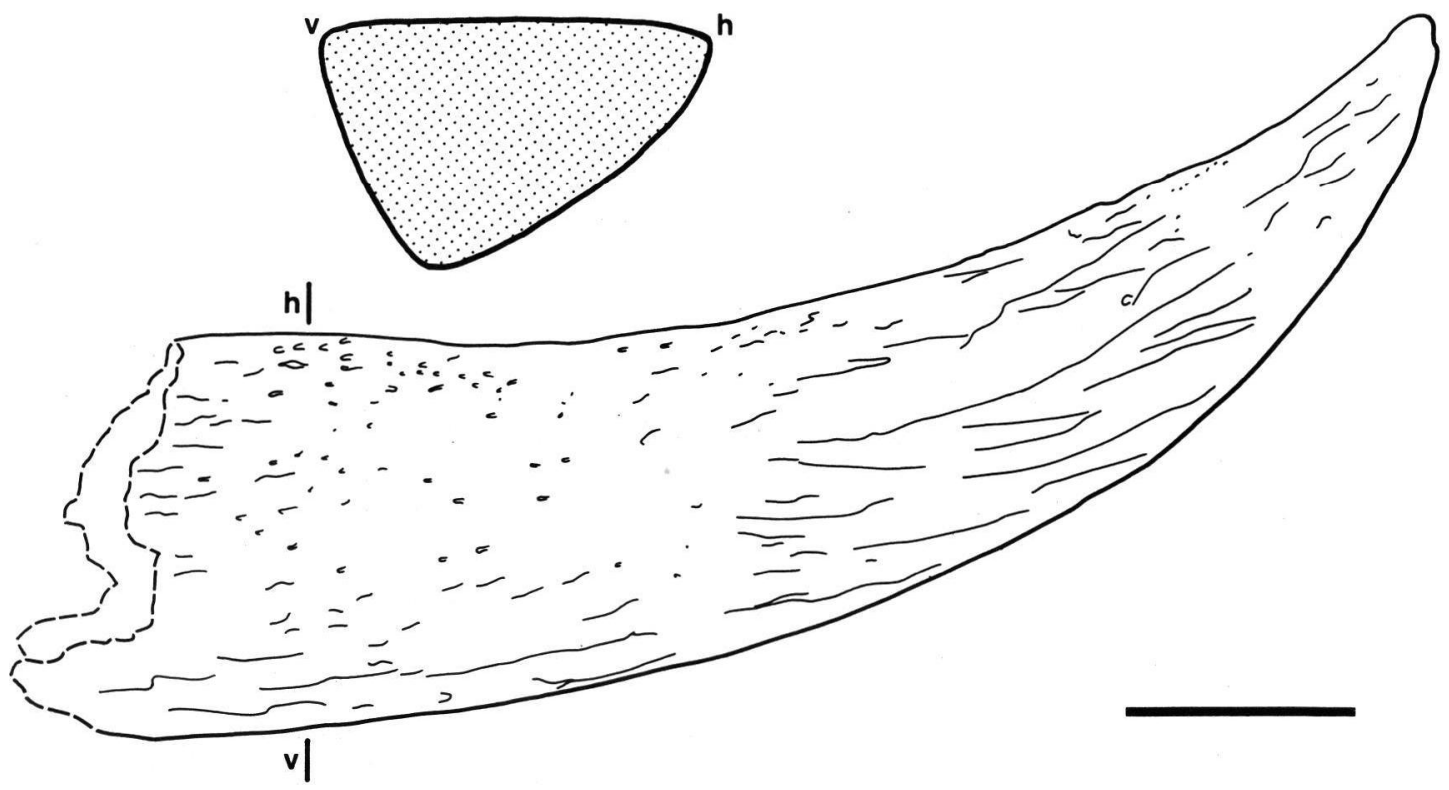

Abb. 6: Hornzapfen von Bubalus murrensis aus Stockstadt/Rhein, mit dem charakteristischen dreieckigen Querschnitt. (Hess. Landesmus. Darmstadt HLMD-RS 2227). Maßstab $=10 \mathrm{~cm}$. 


\subsection{Cervus (Dama) dama}

Damhirschreste sind in den Sand- und Kiesgruben der nördlichen Oberrheinebene unterrepräsentiert, weil die kleinen Knochen in der Regel nicht im großen Fangrost der Kiesgewinnungsanlagen hängen bleiben und im Überkorn nur so stark fragmentiert herauskommen, daß nur wenige Stücke bestimmbar sind. Dennoch wurden in dem betroffenen Gebiet 10 gröBere Geweihfragmente (Abb. 7) und mehrere Skelettelemente aus 4 Gruben gefunden (KoENIGSWALD 1988b).

Für Süddeutschland gab es bislang keine Belege für Cervus dama aus dem letzten Interglazial. Allerdings stammen aus Sachsen und Norddeutschland zahlreiche Funde (BENECKE et al. 1990, SICKENBERG 1969), die auf eine weite Verbreitung dieser Art während des letzten Interglazials schließen lassen.

Cervus dama wird hier unter den „Exoten” aufgeführt, weil die natürliche Fauna des Holozäns den Damhirsch nicht in Mittel- oder Westeuropa kennt. Dagegen tritt Cervus dama in den früheren Interglazialen seit dem Cromerium sensu stricto regelmäßig in diesem Gebiet auf.

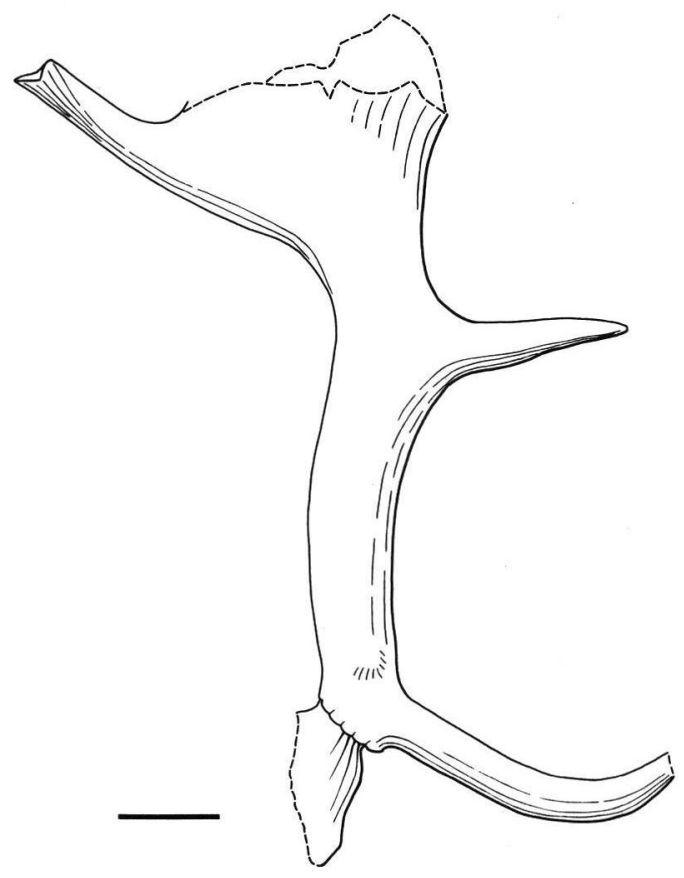

Abb. 7: Schädelechte Geweihstange von Cervus (Dama) dama aus Bobenheim-Roxheim mit dem Ansatz der Schaufel

und der weit abgespreizten Augsprosse.

(Pfalz-Museum, Bad Dürkheim). Maßstab $=5 \mathrm{~cm}$.
Für das Ausbleiben dieser Art im Holozän können klimatische Gründe nicht in erster Linie verantwortlich gemacht werden, denn der Damhirsch wurde im Mittelalter durch den Menschen eingebürgert und hat seitdem recht große Populationen in vielen Teilen Europas bilden können.

Die Ausbreitung des Areals von Säugetieren ist keineswegs nur von klimatischen Faktoren begrenzt. Neben schwer nachzuweisenden Konkurrenzsituationen zu anderen Pflanzenfressern kann auch das Auftreten gewisser Raubtiere die Ausbreitung des Damhirsches eingeschränkt haben. Die jüngste Faunengeschichte Skandinaviens zeigt ein solches Beispiel. Das Reh, Capreolus capreolus, erweiterte sein Areal in den vergangenen Jahrzehnten erheblich nach Norden, ohne daß merkliche Klimaveränderungen zu beobachten waren. Als Grund für die Arealausweitung kommt vielmehr die starke Dezimierung des Wolfes (Canis lupus) durch den Menschen in Betracht (KURTEN 1968).

\subsection{Ein kleines Nashorn}

In den Sanden und Kiesen des letzten Interglazials kommt neben Dicerorbinus kirchbergensis noch ein deutlich kleineres Nashorn vor, das durch mehrere Kiefer und Zähne belegt ist (Abb. 8). Das starke Cingulum der oberen Molaren sowie die geringere Größe kennzeichnen diese Form. Sie zeigt eine gute Übereinstimmung mit einem Fund aus Frankreich von Meyrargues [Bouches-du-Rhone], der stratigraphisch wie die Funde vom Rhein zum letzten Interglazial gestellt wurde (BONIFAY 1961). GUERIN (1980) benutzte für diesen Fund den Namen der altpleistozänen Art Dicerorhinus megarhinus. Die Funde aus der nördlichen Oberrheinebene, auf deren Eigenständigkeit mich M. FORTELIUS aufmerksam machte, werden vorläufig als Dicerorbinus cf. megarbinus bezeichnet, um darauf hinzuweisen, daß hier wohl eine noch unzureichend bekannte jungpleistozäne Nashornart belegt ist.

\section{6 Ökologische Grenzwerte für das Vorkommen der Exoten während des letzten Interglazials in Mitteleuropa}

Bubalus und Hippopotamus haben eine große Affinität zum Wasser, in dem sie viele Stunden verbringen. Auch die rezente Beschränkung auf den tropischsubtropischen Bereich stimmt überein. Damit passen die beiden Gattungen ökologisch gut zusammen, obwohl die eine Gattung nur in Asien, die andere nur in Afrika vorkommt. Es wäre aber voreilig, nach dem Aktualitätsprinzip tropische oder subtropische 
Verhältnisse für Mitteleuropa während jener Zeitabschnitte zu postulieren, in denen Bubalus oder Hippopotamus vorkommen. Bei wesentlich höheren Jahresdurchschnittstemperaturen wären nämlich in der begleitenden Flora und in der dazugehörigen Reptilfauna auch ausgesprochen thermophile Arten zu erwarten. Diese bleiben aber vollständig aus. Die Pollenspektren lassen lediglich eine Erwärmung in den Julitemperaturen um $2-3^{\circ} \mathrm{C}$ gegenüber dem Holozän erkennen (FRENZEL 1989).

Wichtiger als Jahresdurchschnittstemperaturen ist der Klimagang innerhalb eines Jahres. Wie die Tierhaltung in den Zoologischen Gärten zeigt, limitieren nicht die Sommer das Vorkommen von Bubalus oder Hippopotamus. Die Winter bilden die kritische Phase, in denen die Tiere geschützt werden müssen. Allerdings ist mehrfach berichtet worden, daß Flußpferde aus Zirkussen ausgebrochen sind, um im Rhein oder anderen Flüssen zu baden, und das auch in der kühleren Jahreszeit. Aus eigener Anschauung weiß ich, daß im Winter 1984 ein Flußpferd aus einem
Zirkus in Frankfurt a. M. sich freiwillig stundenlang auf einer verschneiten Wiese aufhielt. Der Indische Wasserbüffel (Bubalus arnee), der als Haustier in Siebenbürgen (Rumänien) noch regelmäßig gehalten wird, ist wesentlich empfindlicher. Er bedarf eines sorgfältigen Schutzes vor Zugluft im Stall. Die fossile Form des letzten Interglazials, Bubalus murrensis, steht allerdings dem rezenten Bubalus arnee nicht so nahe, daß man diese Art als Maßstab für die ökologische Toleranz benutzen kann. Einen wichtigen Eckwert gibt aber die starke Affinität beider Gattungen, Bubalus und Hippopotamus, zum Wasser. Es ist undenkbar, daß sie Flußlandschaften besiedelten deren Altwässer im Winter regelmäßig zufroren und mit einer soliden Eisschicht bedeckt waren. Mildere, fast frostfreie Winter werden nicht nur mit einer Erhöhung der Jahresdurchschnittstemperaturen erreicht, sondern ebenso mit einem stärkeren atlantischen Einfluß auf das Klima. Das würde die scheinbare Diskrepanz zwischen Fauna und Flora erklären. Zur Bindung an ein atlantisches Klima paßt, daß Hippopotamus im letzten Interglazial besonders in England in großen Populationen vorgekommen ist.

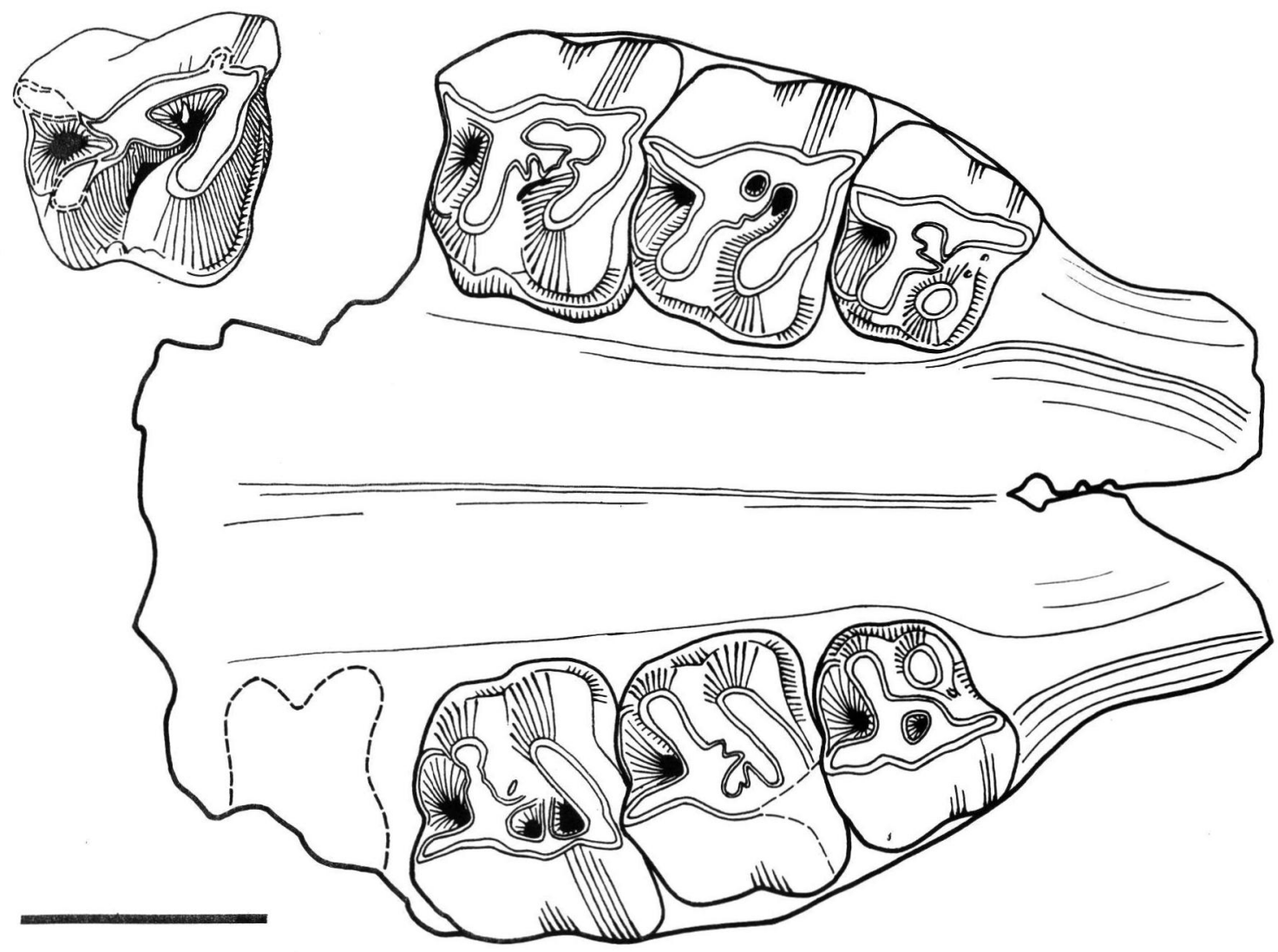

Abb. 8: Oberkieferfragment eines kleinen Nashorns (Dicerorbinus cf. megarbinus) aus Groß-Rohrheim, das auffallend starke Cingula auf der Innenseite der Molaren aufweist.

(Hess. Landesmus. Darmstadt HLMD-RS 2685). Maßstab $=5 \mathrm{~cm}$. 
Für das letzte Interglazial haben RUDDIMAN \& McIINTYRE (1976) besondere ozeanographische Verhältnisse im Nordatlantik nachgewiesen, die sich auch auf das Klima des angrenzenden Festlandteiles ausgewirkt haben dürften. Nach den Foraminiferengesellschaften zu urteilen reichte der Golfstrom weiter nach Norden, womit die Grenze der tropischen Wässer wesentlich weiter nach Norden verschoben wurde, so daß sie einen Teil der westeuropäischen Küste bespülten. In Folge dieser Verschiebung dürften die nordischen Tiefdruckgebiete zurückgehalten worden sein. Größere Feuchtigkeit bei geringer Kälte ist dann für die Winter zu erwarten (freundl. mdl. Mitt. von Prof. Dr. H. FloHN). FRENZEL (1989) nimmt eine durchschnittliche Erhöhung des Niederschlags um etwa $50 \mathrm{~mm}$ an. Damit ist ein wesentlich stärkerer atlantischer Klimaeinfluß zur Zeit der SauerstoffIsotopen Stufe $5 \mathrm{e}$ in West- und Mitteleuropa wahrscheinlich, als er aus den biologischen Anforderungen für das Vorkommen von Bubalus und Hippopotamus gefordert werden müßte.

Ehe man aber versucht ist, derartige klimatische Verhältnisse auch auf die früheren Interglaziale zu übertragen, in denen die Exoten vorgekommen sind, ist es notwendig die Regelmäßigkeit des Vorkommens dieser Arten in den früheren Interglazialen zu untersuchen.

\section{Unterschiedliches Vorkommen von Exoten in den früheren Interglazialen}

Betrachtet man auch die früheren Interglaziale und nicht nur das letzte, dann muß auch der Berberaffe, Macaca sylvanus, zu den Exoten der mitteleuropäischen Interglazialfaunen gezählt werden. Diese westmediterrane Art konnte bisher im letzten Interglazial in Mittel- und Westeuropa nicht nachgewiesen werden. Im Material aus den Kiesgruben der Oberrheinebene könnte zuvor Macaca wegen der geringen Größe leicht übersehen worden sein, aber auch in allen anderen Faunen fehlt jeder Hinweis auf das Vorkommen dieser Art. Das ist um so erstaunlicher, weil Macaca regelmäßig in fast allen früheren Interglazialen seit dem Pliozän nachzuweisen ist [Tegelen, NL (BERNSEN 1931), Hohensülzen, D (FrANZEN 1973), MeiningenUntermaßfeld, D (KAHLKE 1982), West Runton Upper Fresh Water Bed, GB (STUART 1982), Voigtstedt, D (Thenius 1965), Mosbach, D (KoENIGSWALD \& TOBIEN 1987), Bilzingsleben, D (MANIA 1983), Heppenloch, D (ADAM 1975), Swanscombe a, GB (STUART 1982), Hoxn c, GB (STUART 1982), Hunas, D (GroIss 1986)]. Im letzten Interglazial ist dieser Affe weder in Mitteleuropa noch in England belegt und nur spärlich in Italien (ARDITTO \& MOTTURA 1987).
Im Holozän besiedelt Macaca sylvanus Nordafrika und den Felsen von Gibraltar, taucht aber auch im Fossilbericht der Römerzeit und des Mittelalters als importiertes Haustier wieder auf (GULDE 1985; KoCKS 1978).

Tabelle 2: Vorkommen der Exoten

in den pleistozänen Faunen Mittel- und Westeuropas

\section{Hippopo- \\ tamus Bubalus Dama Macaca}

$\begin{array}{lcccc}\text { Holozän } & - & - & - & - \\ \text { letztes Intergl. } & * * * & * * * & * * * & - \\ \text { Ehringsdorf } & - & - & * * * & - \\ \begin{array}{l}\text { Steinheim / } \\ \quad \text { Heppenloch }\end{array} & - & * * * & * * * & * * * \\ \text { Bilzingsleben } & - & - & * * * & * * * \\ \text { Mauer / Mosbach } & * * * & - & * * * & * * * \\ \text { Cromer s.str. } & - & - & * * * & * * * \\ \text { Voigtstedt } & - & - & - & * * * \\ \text { Meiningen } & * * * & - & - & * * *\end{array}$

Vor diesem Hintergrund ist es bemerkenswert (Tab. 2), daß Hippopotamus und Bubalus lediglich im letzten Interglazial zusammen vorkommen, obwohl Hippopotamus dreimal und Bubalus zweimal nach Mitteleuropa vorgedrungen sind. Gerade in diesem Interglazial fehlt Macaca. Wenn nur eine Gattung, Bubalus oder Hippopotamus, vorhanden sind, war das Klima während des Interglazials nicht weniger günstig. Die mittelpleistozänen Interglaziale, in denen nur Macaca in Mitteleuropa vorkam, nicht aber Hippopotamus oder Bubalus, brauchen nicht kühler gewesen zu sein.

Dieses unregelmäßige Auftreten der Exoten in den verschiedenen interglazialen Faunen zeigt, daß man nicht schematisch $z$ wischen besonders warmen Interglazialen mit Exoten und kühleren mit weniger oder gar keinen Exoten unterscheiden kann. Allerdings zeigt das Vorkommen von Bubalus, Hippopotamus und wahrscheinlich auch Macaca einen starken atlantischen Einfluß auf das mitteleuropäische Klima. Allerdings spricht vieles dafür, daß es nicht ausschließlich klimatische Gründe sind, die die Einwanderung während der Interglaziale kontrollieren.

Neben klimatischen Einflüssen begrenzt eine Vielzahl anderer Faktoren die Ausbreitungsmöglichkeiten der 
einzelnen Arten. Es kommen neben geographischen Faktoren vor allem auch Nahrungskonkurrenten und überlegene Raubtiere in Betracht. Daraus ergeben sich einmalige und damit im echten Sinne historische Situationen, deren Ergebnis im Fehlen oder Vorkommen der Arten zwar nachzuweisen sind, deren Faktoren aber schwer zu analysieren sind (KOENIGSWALD $1988 b)$.

\section{Schriftenverzeichnis}

ADAM, K. D. (1965): Neue Flußpferdfunde am Oberrhein. - Jh. geol. Landesamt Baden-Württemberg 7: 621631; Freiburg.

- (1975): Die mittelpleistozäne Säugetier-Fauna aus dem Heppenloch bei Gutenberg (Württemberg). - Stuttgarter Beitr. Naturk., B. 3: 1-247; Stuttgart.

ARBEITSGRUPPE (1980): Hydrogeologische Kartierung und Grundwasserbewirtschaftung Rhein-Neckar-Raum, Analyse des Ist-Zustandes. 71 S. mit Karten, 14 Anlagen; Stuttgart, Wiesbaden-Mainz.

Arditto, G. \& Mottura, A. (1987): An Overview of the Geographic and Chronologic Distribution of West European Cercopithecoids. - Human Evolut. 2: 29-45, $3 \mathrm{Abb}$.

BARTZ, J. (1953): Revision des Bohrprofils der Heidelberger Radium-Sol Therme. Jber. Mitt. oberrh. geol. Ver. N.F. 33: 101-125; Freiburg.

- (1982): Quartär und Jungtertiär II im Oberrheingraben im Großraum Karlsruhe. - Geol. Jb. A 63: 3-237; Hannover.

BeneCKe, N., BÖhme, G., HeINRICH, W. D. (1990): Wirbeltierreste aus interglazialen Beckensedimenten von Gröbern (Kr. Gräfenheinichen) und Grabschütz (Kr. Delitsch). Altenbg. nat.wiss. Forsch. 5: 231-281; Altenburg.

BerNsen, J. J. A. (1931): Eine Revision der Fossilien Säugetierfauna aus den Tonen von Tegelen, III Macacus cf. florentinus COCCHI. - Natuurhist. Maandblad 20: 67-71; Limburg.

BERCKHEMER, F. (1927): Buffelus murrensis n. sp. Ein diluvialer Büffelschädel von Steinheim a.d. Murr. - Jh. Ver. vaterld. Naturkde. Württ. 83: 146-158; Stuttgart.

BeUG, H.-J. (1988): Über die pollenanalytische Datierung einiger jungpleistozäner Proben aus der Oberrheinebene bei Darmstadt. - S. 105-116. - In KoENIGsWALD, W. v. [Hrsg]: Zur Paläoklimatologie des letzten Interglazials im Nordteil der Oberrheinebene. - Paläoklimaforschung 4; Stuttgart-New York (G. Fischer).

BonifaY, M.-F. (1961): Étude des restes de Rhinocéros de Merck provenant de Meyrargues (Bouches-du-Rhone). - Ann. Pal. 47: 77-89; Paris.
EIsSmanN, L. [Hrsg.] (1990): Die Eemwarmzeit und die Frühe Weichselkaltzeit im Saale-Elbe-Gebiet: Geologie, Paläontologie, Palökologie. Ein Beitrag zum jüngeren Quartär in Mitteleuropa. - Altenburger Naturwiss. Forsch. 5: 1-301; Altenburg.

FAHLBUSCH, K. (1975): Störungszonen an den Rheingraben-Schultern (Hardt, Darmstadt) mit Hinweisen auf junge Bewegungen. - Notizbl. hess. L.-Amt Bodenforsch. 103: 315-326; Wiesbaden.

FranZEN, J. L. (1973): Ein Primate aus den altpleistozänen Schneckenmergeln von Hohensülzen (Rheinhessen). Senckenbergiana lethaea 54: 345-358; Frankfurt a. M.

- (1978): New discovery of Bubalus from the late Pleistocene of Western Germany. - II. Congr. theorio. internat., Abstr.: 99; Brünn.

- \& KoENIGSWALD, W. v. (1979): Erste Funde vom Wasserbüffel (Bubalus murrensis) aus pleistozänen Schottern des nördlichen Oberrhein-Grabens. - Senckenbergiana lethaea 60 (1/3): 253-263); Frankfurt a. M. - In KoENIGSWALD W. v. [Hrsg.]: Zur Paläoklimatologie des letzten Interglazials im Nordteil der Oberrheinebene. - Paläoklimaforschung 4; Stuttgart-New York (G. Fischer).

FrenZel, B. (1989): Projektgruppe „Terrestrische Paläoklimatologie" im Rahmen des nationalen Klimforschungsprogramms der Bundesregierung. - 453-461 - Akademie der Wissenschaften und der Literatur Mainz 1949-1989. - Stuttgart (Steiner).

Grolss, J. T. (1986): Erste Funde von Primaten in der Höhlenruine von Hunas / Hartmannshof (Lkrs. Nürnberger Land). - Altnürnberger Landschaft 35/2: 193197; Nürnberg.

GueriN, C. (1980): Les rhinoceros (Mammalia, Perissodactyla) du Miocéne terminal au Pleistocéne supérieur en Europe occidentale. Comaraison avec les espéces actuelles. - Docu., Lab. Géol. Lyon, 79: 1-185; Lyon.

GuLDE, V. (1985): Osteologische Untersuchungen an Tierknochen aus dem römischen Vicus Rainau-Buch (Ostalbkreis). - Materialh. z. Vor- u. Frühgesch. in BadenWürttemberg 5; Stuttgart.

KAHLKE, H. D. (1982): Hippopotamus antiquus DESMARET, 1822 aus dem Pleistozän von Meiningen in Südthüringen (Bezirk Suhl DDR). —Z. geol. Wiss. 10: 943-949; Berlin.

KAHLKE, R.-D. (1985): Altpleistozäne Hippopotamus-Reste von Untermaßfeld bei Meiningen (Bezirk Suhl DDR) (vorläufige Mitteilung). - Säugetierkd. Inf. 2: 227233; Jena.

- (1989): Die unterpleistozänen Hippopotamus-Reste von Würzburg-Schalksberg. - Quartär 39/40: 67-94; Bonn.

- (1990): Zum Stand der Erforschung fossiler Hippopotamiden (Mammalia, Artiodacyla), eine Übersicht. Quartärpaläontologie 8: 107-118; Berlin. 
Kocks, B.-M. (1978): Die Tierknochen aus den Burgen auf dem Weinberg in Hitzacker/Elbe und in Dannenberg (Mittelalter) - I. Die Nichtwiederkäuer. - Diss. Univ. München.

KOENIGSWALD, W. v. (1986a): Ein Skelett vom Waldelefanten aus den jungpleistozänen Rheinschottern von Crumstadt bei Darmstadt. - Cranium 3/1: 8-13; Dieren.

- (1986b): Bezichungen des pleistozänen Wasserbüffels (Bubalus murrensis) aus Europa zu den asiatischen Wasserbüffeln. - Z. Säugetierk. 51: 312-323; Hamburg.

- [Hrsg.] (1988a): Zur Paläoklimatologie des letzten Interglazials im Nordteil der Oberrheinebene. - Paläoklimaforschung 4: 1-327; Stuttgart-New York (G. Fischer).

- (1988b): Paläoklimatische Aussage letzinterglazialer Säugetiere aus der nördlichen Oberrheinebene. - S. 205-314. - In KOENIGSWALD, W. v. [Hrsg.]: Zur Paläoklimatologie des letzten Interglazials im Nordteil der Oberrheinebene. - Paläoklimaforschung 4; Stuttgart-New York (G. Fischer).

— \& Beug, H. J. (1988): Schlußbetrachtung. - 321327. - In Koenigswald W. v. [Hrsg.]: Zur Paläoklimatologie des letzten Interglazials im Nordteil der Oberrheinebene. - Paläoklimaforschung 4; StuttgartNew York (G. Fischer).

- \& LÖSCHER, M. (1982): Jungpleistozäne Hippopotamus-Funde aus der Oberrheinebene und ihre biogeographische Bedeutung. - N. Jb. Geol. Paläont. Abh. 163: 331-348; Stuttgart.

— \& ToBien, H. (1987): Bemerkungen zur Altersstellung der pleistozänen Mosbach-Sande bei Wiesbaden. Geol. Jb. Hessen 115: 227-237; Wiesbaden.

KOLFSCHOTEN, T. v. (1990): The evolution of the mammal fauna in the Netherlands and the Middle Rhine area (Western Germany) during the late middle Pleistocene. - Meded. Rijks. Geol. Dienst. 43/3: 1-69; Haarlem.

KreuZ, A. \& Leistikow, K. U. (1988): Holzfunde aus jungquartären Ablagerungen der nördlichen Oberrheinebene und ihre chronostratigraphische Deutung. - S. 117148. - In KoENigswaLD, W. v. [Hrsg.]: Zur Paläoklimatologie des letzten Interglazials im Nordteil der Oberrheinebene. - Paläoklimaforschung 4; StuttgartNew York (G. Fischer).

Kuss, S. E. (1957): Altpleistozäne Reste des Hippopotamus antiquus DESMAREST vom Niederrhein. - Jh. geol. Landesamt Baden-Württemberg 2: 299-331; Freiburg i. Br.

KURTEN, B. (1968): Pleistocene mammals of Europe. 316 S.; London (Weidenfeld and Nicolson).

LOSCHER, M. (1981): Die stratigraphische Gliederung des Jungpleistozäns im Neckarschwemmfächer bei Heidelberg. - Aufschluß 32: 191-199; Heidelberg.
LOSCHER, M. (1988): Stratigraphische Interpretation der jungpleistozänen Sedimente in der Oberrheinebene zwischen Bruchsal und Worms. - S. 79-104. - In KOENIGSWALD, W. v. [Hrsg.]: Zur Paläoklimatologie des letzten Interglazials im Nordteil der Oberrheinebene. - Paläoklimaforschung 4; Stuttgart-New York (G. Fischer).

-, Becker, B., Bruns, M., Hieronymus, U., Mäusbacher, R., MÜNNICH, M., MUUNZING K. \& SCHNEIDER, J. (1980): Neue Ergebnisse über das Jungquartär im Neckarschwemmfächer bei Heidelberg. - Eiszeitalter u. Gegenwart. 30: 89-100; Hannover.

MANIA, D. (1983): Zum Stand der Untersuchungen weiterer Fossilgruppen aus dem Travertin von Bilzingsleben. 157-163. - In MaI, D. H., Mania, D., NÖ́ZZOLd, T., TOepfer, V., Vlcek, E. \& Heinrich, W. D.: Bilzingsleben II, Homo erectus - seine Kultur und seine Umwelt. - Veröff. Landesmus. Vorgesch. Halle 36: 1-258; Berlin.

Martin, Th. (1987): Artunterschiede an den Langknochen großer Artiodactyla des Jungpleistozäns Mitteleuropas. - Cour. Forsch.-Inst. Senckenberg 96: 1-124; Frankfurt a. M.

- (1990): Jungpleistozäne und holozäne Skelettfunde von Bos primigenius und Bison priscus aus Deutschland und ihre Bedeutung für die Zuordnung isolierter Langknochen. - Eiszeitalter u. Gegenwart 40: 1-19; Hannover.

MERCK, J. H. (1783): Über den Ursprung der Fossilien in Teutschland. - Der Teutsche Mekur 1783: 50-63; Weimar.

NeUFER, F. O. \& IGEL, W. (1983): Ein Wasserbüffel-Fund aus pleistozänen Schottern bei Eich (nördlicher Oberrheingraben). Mainzer Naturw. Archiv. 21: 187-197; Mainz.

SCHeER, H.-D. (1976): Die pleistozänen Flußterrassen in der östlichen Mainebene. - Geol. Jb. Hessen 104: 61-86; Wiesbaden.

Ruddiman, W. F. \& McInTyre, A. (1976): North-east Atlantic paleoclimatic changes over the past 600000 years. - Mem. geol. Soc. Amer. 145: 111-146.

RutTe, E. (1968): Die Cromer-Wirbeltierfundstelle Würzburg-Schalksberg. - Abh. naturwiss. Ver. Würzburg 8: 1-26; Würzburg.

SCHERTZ, E. (1937): Ein neuer Wasserbüffel aus dem Diluvium Mitteldeutschlands (Buffelus wanckeli nov. spec.) — Paläont. Z. 19: 57-72; Berlin.

Sсносн, W. H. (1988): Holzfunde aus den jungpleistozänen Sedimenten der Oberrheinebene. - S. 149-172. - In Koenigswald, W. v. [Hrsg.]: Zur Paläoklimatologie des letzten Interglazials im Nordteil der Oberrheinebene. - Paläoklimaforschung 4; Stuttgart-New York (G. Fischer). 
SCHWEISS, D. (1988): Jungpleistozäne Sedimentation in der nördlichen Oberrheinebene. - S. 19-78. - In KOENIGSWALD, W. v. [Hrsg.]: Zur Paläoklimatologie des letzten Interglazials im Nordteil der Oberrheinebene. Paläoklimaforschung 4; Stuttgart-New York (G. Fischer).

SICKENBERG, O. (1969): Die Säugetierfauna der Kalkmergel von Lehringen (Krs. Verden/Aller) im Rahmen der Eemzeitlichen Faunen Nordwestdeutschlands. - Geol. Jb. 87: 551-564; Hannover.

Stampfl, H. R. (1982): Die Geschichte des Wilhelm Fabri an die Berner Bibliothek - Jb. Bernisch. Museum 61/62: 47-97; Bern.

StUART, A. J. (1983): Pleistocene Vertebrates in the British Isles. 1-212; London (Longman).

- (1986): Pleistocene occurrences of Hippopotamus in Britain. - Quartärpaläontologie 6: 209-218; Berlin.
Sutcliffe, A. J. (1959): The Hippopotamus in Britain. Bull. Mammal. Soc. British Isles 11: 36-40; London.

Sutcuiffe, A. J. (1985): On the Track of Ice Age Mammals. - 1-224; London (British Museum).

ThenIUS, E. (1962): Die Großsäugetiere des Pleistozäns in Mitteleuropa. - Z. F. Säugetierk. 27: 65-83; Hamburg.

- (1965): Ein Primatenrest aus dem Altpleistozän von Voigtstedt in Thüringen. Paläont. Abh. A2(2/3): 682-686; Berlin.

WIEGANK, F. (1982): Ergebnisse magnetostratigraphischer Untersuchungen im höheren Känozoikum der DDR. Z. geol. Wiss. 10: 737-744; Berlin.

Manuskript eingegangen am 25. 10. 1990 